\title{
Evaluation of the effects of Angelicae dahuricae radix on the morphology and viability of mesenchymal stem cells
}

\author{
SU-HYEON JEONG ${ }^{1}$, BO-BAE KIM ${ }^{2}$, JI-EUN LEE ${ }^{2}$, YOUNGKYUNG KO ${ }^{2}$ and JUN-BEOM PARK ${ }^{2}$ \\ ${ }^{1}$ Department of Rehabilitation Medicine of Korean Medicine, Chungju Hospital of Korean Medicine, \\ College of Korean Medicine, Semyung University, Jecheon-si, Chungcheongbuk-do 390-711; \\ ${ }^{2}$ Department of Periodontics, College of Medicine, The Catholic University of Korea, \\ Seoul 137-701, Republic of Korea
}

Received June 6, 2014; Accepted February 20, 2015

DOI: $10.3892 / \mathrm{mmr} .2015 .3456$

\begin{abstract}
Angelicae dahuricae radix is a traditional herbal medicine used to treat various diseases in China and Korea, such as colds, headaches, rhinitis and psoriasis. Angelicae dahuricae radix has been used as an anti-inflammatory, analgesic, antipyretic and antioxidant remedy. This study was performed in order to evaluate the effects of the extracts of Angelicae dahuricae radix on the morphology and viability of mesenchymal stem cells derived from the gingiva. Mesenchymal stem cells derived from the gingiva were grown in the presence of Angelicae dahuricae radix at final concentrations that ranged from 0.001 to $100 \mu \mathrm{g} / \mathrm{ml}$. The morphology of the cells was viewed under an inverted microscope, and the analysis of cell proliferation was performed with cell counting kit-8 (CCK-8) on days 1,3 and 7 . The cells in the control group had spindle-shaped, fibroblast-like morphology at days 1, 3 and 7 under optical microscopy. The shapes of the cells in $0.001,0.01,0.1,1,10$ and $100 \mu \mathrm{g} / \mathrm{ml}$ Angelicae dahuricae radix were similar to the shapes of the cells in the control group. The relative values of the CCK-8 assays of $0.001,0.01,0.1,1,10$, and $100 \mu \mathrm{g} / \mathrm{ml}$ Angelicae dahuricae radix were $102.5 \pm 0.6,133.3 \pm 9.6,148.4 \pm 20.5$, $147.7 \pm 12.6,132.3 \pm 27.7$ and $101.1 \pm 4.6 \%$, respectively, when the CCK- 8 result of the control group on day 1 was considered to be $100 \%$. There was a marginal increase in cell proliferation at 0.1 and $1 \mu \mathrm{g} / \mathrm{ml}$ groups at day 1 ; however, this did not achieve statistical significance $(\mathrm{P}=0.052)$. The
\end{abstract}

Correspondence to: Dr Jun-Beom Park, Department of Periodontics, Seoul St. Mary's Hospital, College of Medicine, The Catholic University of Korea, 222 Banpo-daero, Seocho-gu, Seoul 137-701, Republic of Korea

E-mail: jbassoonis@yahoo.co.kr

Key words: cell shape, cell survival, herbal medicine, plant roots, stem cells relative values of the CCK-8 assays of $0.001,0.01,0.1,1,10$ and $100 \mu \mathrm{g} / \mathrm{ml}$ Angelicae dahuricae radix were $96.5 \pm 1.3$, $89.3 \pm 0.9,90.3 \pm 3.0,84.8 \pm 12.2,92.3 \pm 4.5$ and $86.8 \pm 11.7 \%$, respectively, when the CCK- 8 result of the control group on day 3 was considered to be $100 \%(\mathrm{P}>0.05)$. The relative values of the CCK- 8 assays of $0.001,0.01,0.1,1,10$ and $100 \mu \mathrm{g} / \mathrm{ml}$ Angelicae dahuricae radix day 7 were $94.9 \pm 22.3,102.8 \pm 22.1,127.4 \pm 7.4,130.4 \pm 1.3,129.2 \pm 10.8$ and $124.8 \pm 9.1 \%$, respectively, when the CCK- 8 result of the control group on day 7 was considered to be $100 \%$, but there were no statistically significant differences among the groups $(\mathrm{P}>0.05)$. Within the limits of this study, Angelicae dahuricae radix at the tested concentrations did not produce statistically significant differences in the viability of stem cells derived from the gingiva.

\section{Introduction}

Angelicae dahuricae is a perennial plant that grows naturally throughout large areas of China. It has a strong scent, and its leaves are used to make incense (1). Angelicae dahuricae radix is the dried root of Angelica dahurica Bentham et Hooker and Angelica dahurica (Fisch. ex Hoffm). Benth. et Hook. f. var. formosana (Boiss). Shan et Yuan, known as Bai Zhi in Chinese, and is used in traditional Chinese medicine to treat various diseases (2).

Angelicae dahuricae radix has been used for the treatment of colds, headaches, rhinitis and psoriasis in traditional medicine (3). Research has been performed on the anti-inflammatory, analgesic, antipyretic, antioxidant and cytochrome P450 activity of Angelicae dahuricae radix (4-6). Furthermore, Angelicae dahuricae radix has been suggested for use in the treatment of oral diseases, including toothache (3). Limited information is currently available regarding the effects of Angelicae dahuricae radix on dental tissue, and there is no information on its effects on the mesenchymal stem cells derived from the gingiva.

The aim of the present study was to evaluate the effects of extracts of Angelicae dahuricae radix on the morphology and viability of human stem cells derived from the gingiva. To the 

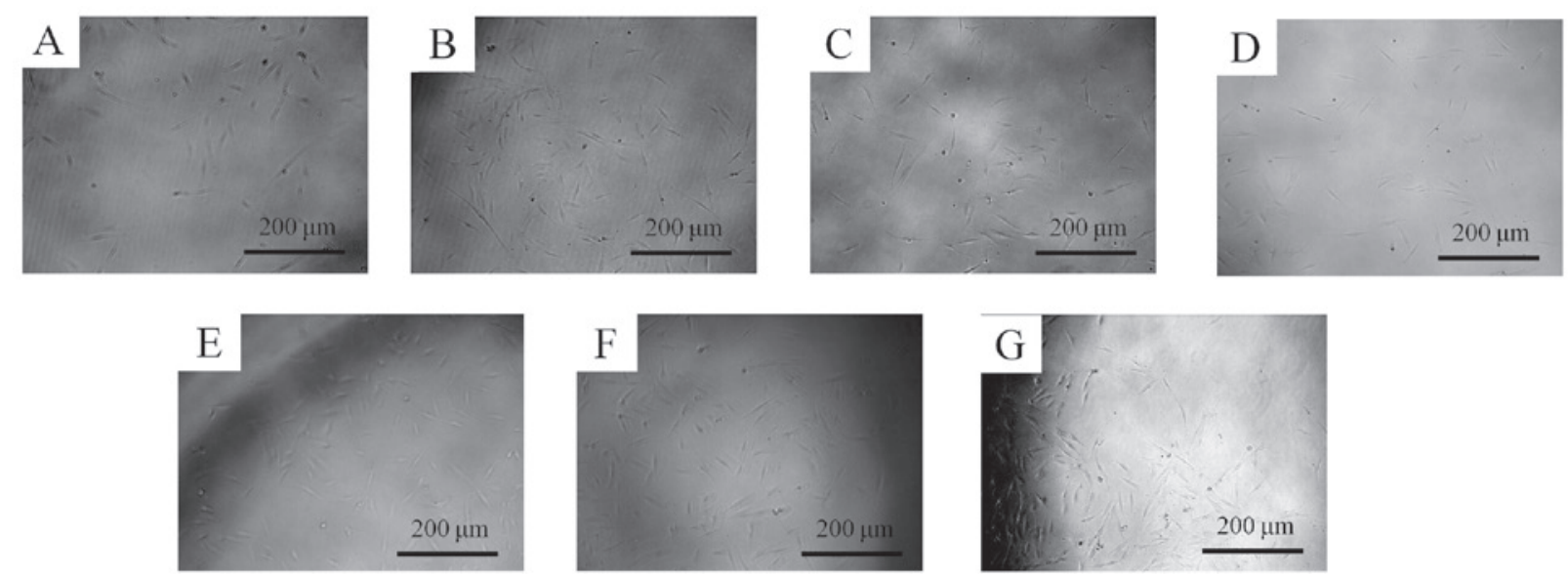

Figure 1. Evaluation of cell morphology on day 1 following treatment with different concentrations of Angelicae dahuricae radix. (A) Control, (B) $0.001 \mu \mathrm{g} / \mathrm{ml}$ group, (C) $0.01 \mu \mathrm{g} / \mathrm{ml}$ group, (D) $0.1 \mu \mathrm{g} / \mathrm{ml}$ group, (E) $1 \mu \mathrm{g} / \mathrm{ml}$ group, (F) $10 \mu \mathrm{g} / \mathrm{ml}$ group and (G) $100 \mu \mathrm{g} / \mathrm{ml}$ group.

best of our knowledge, this investigation is the first to elucidate the effect of Angelicae dahuricae radix on stem cells derived from the gingiva.

\section{Materials and methods}

Preparation of the materials. The dry roots of Angelica dahurica Bentham et Hooker (500 g) were immersed in 2,000 $\mathrm{ml}$ distilled water for $2 \mathrm{~h}$ and boiled under reflux for $2 \mathrm{~h} 30 \mathrm{~min}$. The resulting extract was centrifuged at 5,000 x g for $10 \mathrm{~min}$. The supernatant was concentrated to $300 \mathrm{ml}$ using a rotary evaporator under reduced pressure (Eyela NE-1001, Tokya Rikakikai Co. Ltd., Tokyo, Japan). The concentrates were then freeze-dried in a lyophilizer (Labconco, Kansas, MO, USA) to obtain $182.5 \mathrm{~g}$ of solid residue, resulting in a yield of $36.5 \%(\mathrm{w} / \mathrm{w})$.

Isolation and culture of the stem cells derived from the gingiva. Healthy gingival tissues were obtained from four healthy patients undergoing crown-lengthening procedures. This study was reviewed and approved by the Institutional Review Board of Seoul St. Mary's Hospital, College of Medicine, The Catholic University of Korea (Seoul, Republic of Korea; KC11SISI0348), and informed consent was obtained from all participants.

The tissues were immediately placed in sterile phosphate-buffered saline (PBS, Welgene, Daegu, Korea) with $100 \mathrm{U} / \mathrm{ml}$ penicillin and $100 \mu \mathrm{g} / \mathrm{ml}$ streptomycin (Sigma-Aldrich, St. Louis, MO, USA) at $4^{\circ} \mathrm{C}$. The gingival tissue was de-epithelialized, minced, digested with collagenase IV (Sigma-Aldrich) and incubated at $37^{\circ} \mathrm{C}$ in a humidified incubator with $5 \% \mathrm{CO}_{2}$ and $95 \% \mathrm{O}_{2}$. The non-adherent cells were washed with PBS after $24 \mathrm{~h}$, replaced with fresh medium, and fed every 2-3 days.

Evaluation of stem cell morphology. The stem cells were plated at a density of $2.0 \times 10^{3}$ cells/well in 96 -well plates The cells were incubated in minimum Essential medium $\alpha$ ( $\alpha$-MEM, Gibco, Grand Island, NY, USA) that was composed of $15 \%$ fetal bovine serum (Gibco), $100 \mathrm{U} / \mathrm{ml}$ penicillin and $100 \mu \mathrm{g} / \mathrm{ml}$ streptomycin (Sigma-Aldrich),
$200 \mathrm{mM}$ L-Glutamine (Sigma-Aldrich) and $10 \mathrm{mM}$ ascorbic acid 2-phosphate (Sigma-Aldric) in the presence of the Angelicae dahuricae radix at final concentrations that ranged from 0.001 to $100 \mu \mathrm{g} / \mathrm{ml}$ [0 (control), $0.001,0.01,0.1,1,10$, and $100 \mu \mathrm{g} / \mathrm{ml}]$. The morphology of the cells was viewed under an inverted microscope (Leica DM IRM, Leica Microsystems, Wetzlar, Germany) on days 1,3 and 7. The images were saved as JPEG files.

Determination of cell proliferation. The analysis of cell proliferation was performed on days 1, 3 and 7. Viable cells were identified using a cell counting kit-8 (CCK-8, Dojindo, Tokyo, Japan) assay. The spectrophotometric absorbance was measured with a microplate reader (BioTek, Winooski, VT, USA), and the analysis was performed in triplicate.

Statistical analysis. The findings are represented as the mean \pm standard deviation of the experiments. Analysis of normality was performed, and a one-way analysis of variance (ANOVA) with post hoc test was performed to determine the differences between the groups using a commercially available program (SPSS 12 for Windows, SPSS Inc., Chicago, IL, USA). $\mathrm{P}<0.05$ was considered to indicate a statistically significant difference.

\section{Results}

Evaluation of cell morphology. The morphology of the stem cells at day 1 is shown in Fig. 1. Under optical microscopy, the control group cells had a spindle-shaped, fibroblast-like morphology. The shapes of the cells treated with 0.001,0.01,0.1, 1, 10, and $100 \mu \mathrm{g} / \mathrm{ml}$ Angelicae dahuricae radix were similar to the shapes of the cells in the control group. The morphology of the cells on day 3 is shown in Fig. 2. The shapes of the cells treated with $0.001,0.01,0.1,1,10$, and $100 \mu \mathrm{g} / \mathrm{ml}$ were similar to those of cells in the control group. No significant alterations were noted in the treated groups ( 0.001 to $100 \mu \mathrm{g} / \mathrm{ml}$ groups) when compared with the control group. The morphology of the cells on day 7 is shown in Fig. 3. The shapes of the cells treated with $0.001,0.01,0.1,1,10$, and $100 \mu \mathrm{g} / \mathrm{ml}$ groups were similar to the shapes of the cells in the untreated control group. 

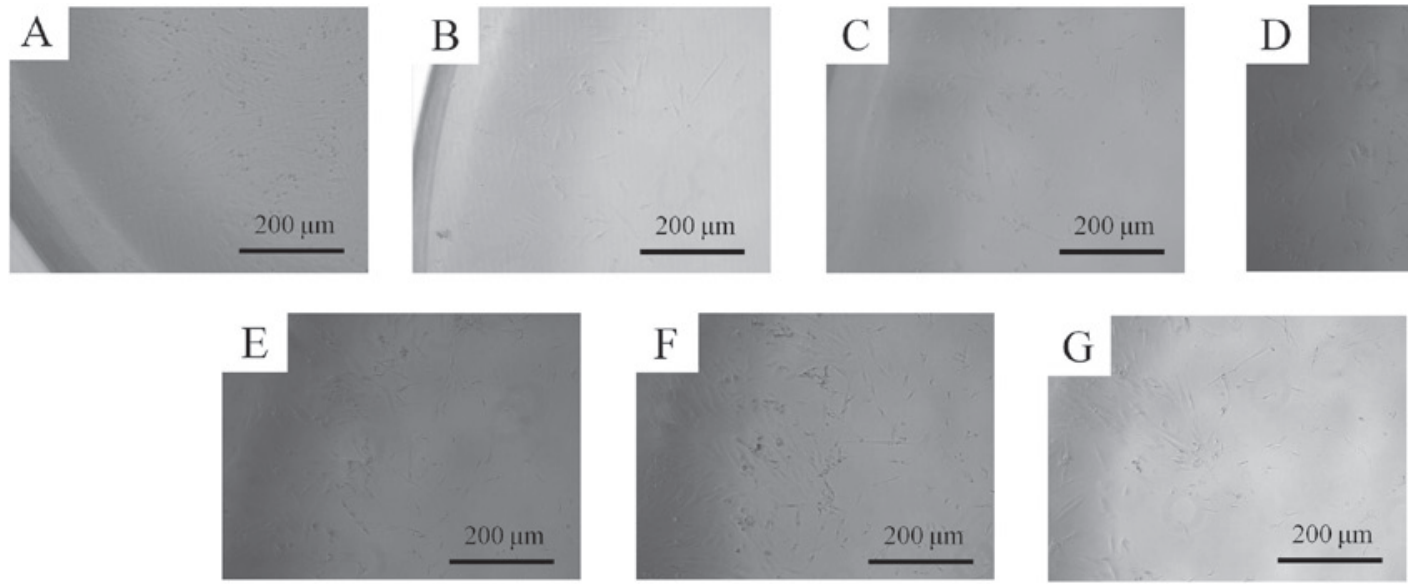

Figure 2. Cell morphology on day 3 following treatment with different concentrations of Angelicae dahuricae radix. (A) Control, (B) $0.001 \mu \mathrm{g} / \mathrm{ml}$ group, (C) $0.01 \mu \mathrm{g} / \mathrm{ml}$ group, (D) $0.1 \mu \mathrm{g} / \mathrm{ml}$ group, (E) $1 \mu \mathrm{g} / \mathrm{ml}$ group, (F) $10 \mu \mathrm{g} / \mathrm{ml}$ group and (G) $100 \mu \mathrm{g} / \mathrm{ml}$ group.
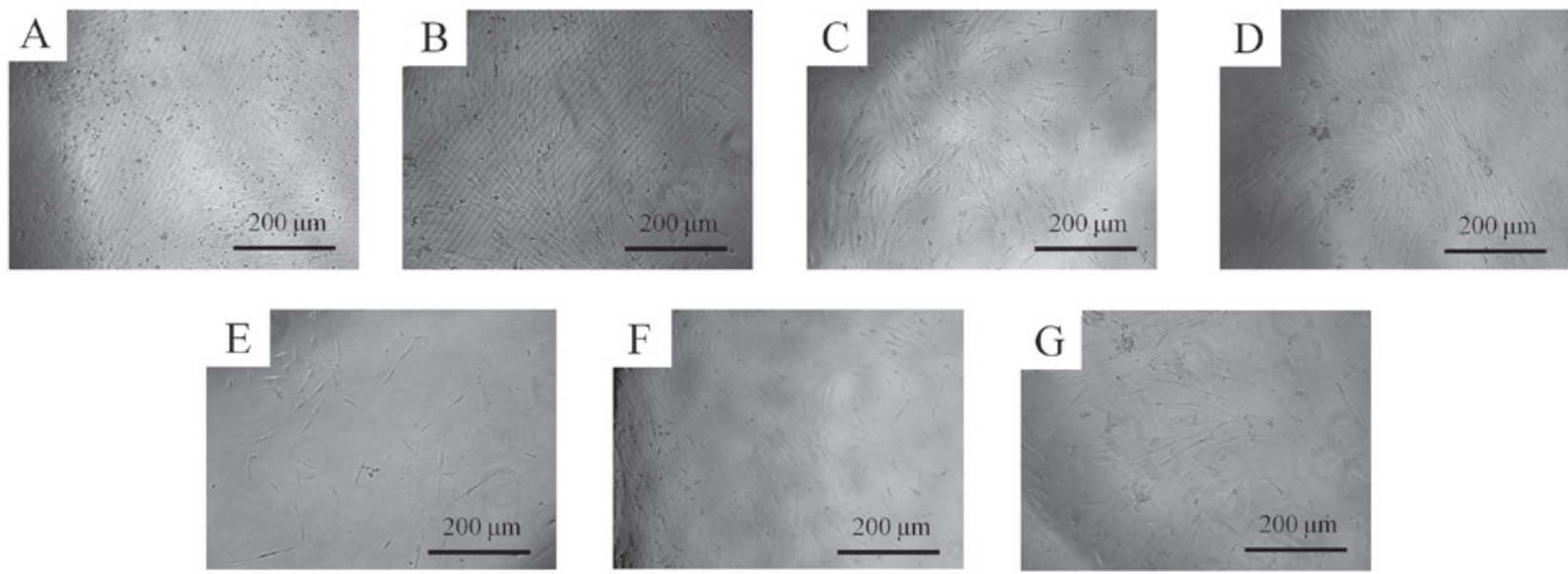

Figure 3. Cell morphology on day 7 following treatment with different concentrations of Angelicae dahuricae radix. (A) Control, (B) $0.001 \mu \mathrm{g} / \mathrm{ml}$ group, (C) $0.01 \mu \mathrm{g} / \mathrm{ml}$ group, (D) $0.1 \mu \mathrm{g} / \mathrm{ml}$ group, (E) $1 \mu \mathrm{g} / \mathrm{ml}$ group, (F) $10 \mu \mathrm{g} / \mathrm{ml}$ group and (G) $100 \mu \mathrm{g} / \mathrm{ml}$ group.

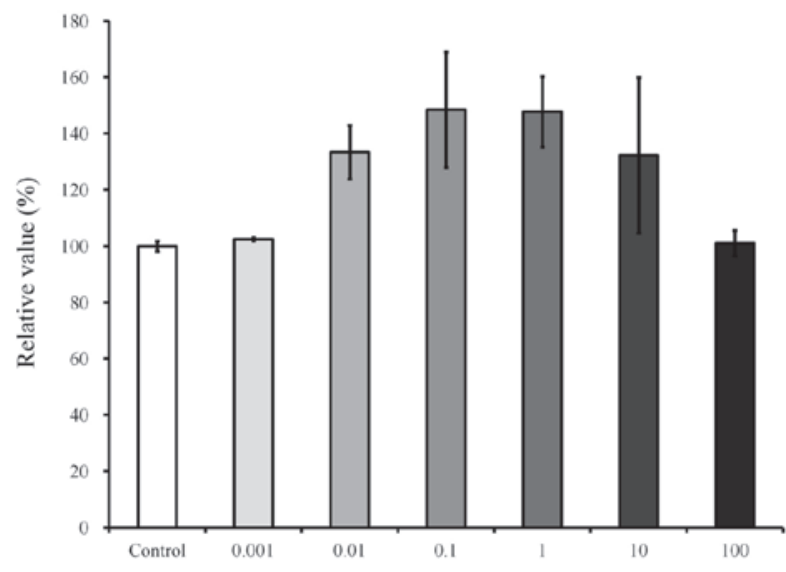

Figure 4. Cellular viability on day 1 following treatment with different concentrations of Angelicae dahuricae radix.

Cell proliferation. The results of cell proliferation at day 1 are shown in Fig. 4, respectively. The relative values of the CCK-8 assays of $0.001,0.01,0.1,1,10$, and $100 \mu \mathrm{g} / \mathrm{ml}$ Angelicae dahuricae radix were $102.5 \pm 0.6$, $133.3 \pm 9.6 \%, 148.4 \pm 20.5,147.7 \pm 12.6,132.3 \pm 27.7$ and

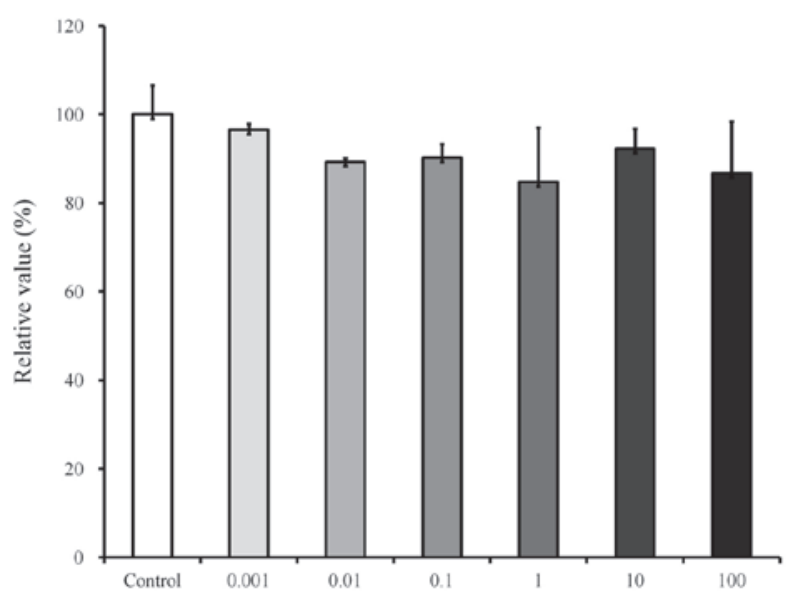

Figure 5. Cellular viability on day 3 following treatment with different concentrations of Angelicae dahuricae radix.

$101.1 \pm 4.6 \%$, respectively, when the CCK- 8 result of the control group on day 1 was considered to be $100 \%(100.0 \pm 1.8)$. The proliferation rate of the cultures that were growing in the presence of Angelicae dahuricae radix increased marginally 


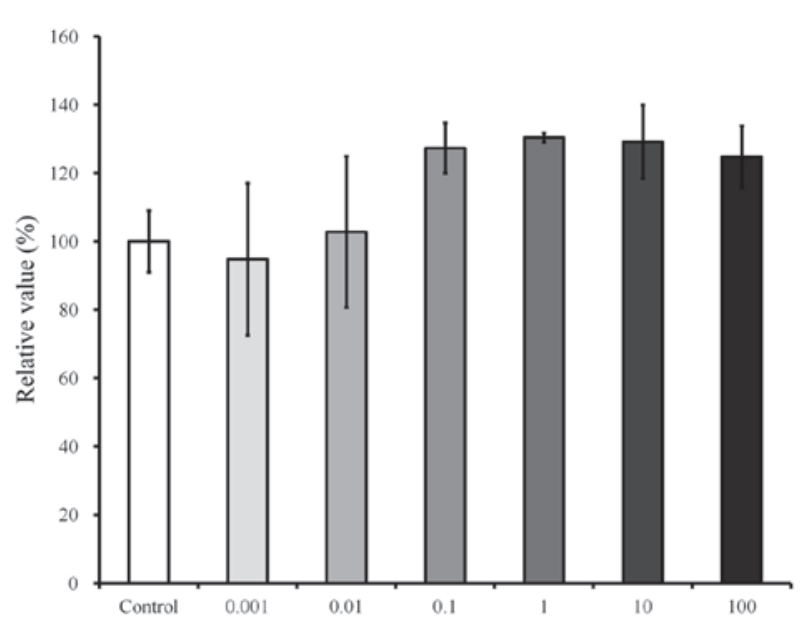

Figure 6. Cellular viability on day 7 following treatment with different concentrations of Angelicae dahuricae radix.

in the 0.1 and $1 \mu \mathrm{g} / \mathrm{ml}$ groups, but this was not indicated to be statistically significant $(\mathrm{P}=0.052)$.

The results at day 3 are shown in Fig. 5. The relative values of the CCK- 8 assays of $0.001,0.01,0.1,1,10$, and $100 \mu \mathrm{g} / \mathrm{ml}$ of Angelicae dahuricae radix were $96.5 \pm 1.3,89.3 \pm 0.9,90.3 \pm 3.0$, $84.8 \pm 12.2,92.3 \pm 4.5$ and $86.8 \pm 11.7 \%$, respectively, when the CCK-8 result of the control group on day 3 was considered to be $100 \%(100.0 \pm 6.5)$. No significant differences were noted among the groups $(\mathrm{P}>0.05)$.

The results at day 7 are shown in Fig. 6. The relative values of the CCK-8 assays of $0.001,0.01,0.1,1,10$, and $100 \mu \mathrm{g} / \mathrm{ml}$ of Angelicae dahuricae radix were 94.9 $\pm 22.3,102.8 \pm 22.1$, $127.4 \pm 7.4,130.4 \pm 1.3,129.2 \pm 10.8$ and $124.8 \pm 9.1 \%$, respectively, when the CCK-8 result of the control group on day 7 was considered to be $100 \%(100.0 \pm 9.0)$. The cultures that were growing in the presence of Angelicae dahuricae radix exhibited marginally increased proliferation in the 1 and $10 \mu \mathrm{g} / \mathrm{ml}$ groups; however, this was not observed to achieve statistical significance $(\mathrm{P}>0.05)$.

\section{Discussion}

This study discussed the effects of Angelicae dahuricae radix on the morphology and proliferation of the human mesenchymal stem cells derived from periodontal tissue. This study showed that cell proliferation of the stem cells were influenced by Angelicae dahuricae radix at day 1 and a marginal increase in cell number was observed at certain concentrations $(0.1$ to $1 \mu \mathrm{g} / \mathrm{ml})(\mathrm{P}=0.052)$; however, a significant increase in cell proliferation was not achieved at day 3 and 7.

Angelicae dahuricae radix is a traditional herbal medicine used to treat various diseases. More recently, it was reported that Angelicae dahuricae radix suppressed the development of atopic dermatitis-like skin lesions and could be used for the treatment of hypertension and asthma $(3,7,8)$. A number of analytical methods have been reported for the determination of several coumarins in Angelicae dahuricae radix, including capillary electrochromatography, high-performance liquid chromatography, liquid chromatography-mass spectrometry, gas chromatography-mass spectrometry and high-speed countercurrent chromatography (7,9-12). It appears that the crude extract and essential oils of Angelicae dahuricae radix may include multiple potentially active chemical compounds. The main components of Angelicae dahuricae radix are reported to be volatile oil and ingredients of coumarin, including imperatorin, isoimperatorin and cnidilin, and these active ingredients of Angelicae dahuricae radix may explain its multiple functions (13). Previous studies have shown that auraptenol, a major coumarin component from Angelicae dahuricae radix, had a robust antinociceptive effect in a chronic neuropathic pain mouse model (1).

Limited studies were performed to evaluate the effects of Angelicae dahuricae radix on the cell viability in vitro and in vivo $(14,15)$, and Angelicae dahuricae radix extract showed cytotoxicity toward cancer cells, including mouse lymphocytic leukemia cells, human promyelocytic leukemia cells, human myelogenous leukemia cells, and mouse melanoma cells (14). Coumarins were isolated from Angelicae dahuricae radix by silica gel column chromatography, and the half maximal inhibitory concentration $\left(\mathrm{IC}_{50}\right)$ values varied from 8.6 to $14.6 \mu \mathrm{g} / \mathrm{ml}$ cells. However, another study showed that extracts of Angelicae dahuricae radix combined with methanol, water and ethyl acetate at $100 \mu \mathrm{g} / \mathrm{ml}$ did not produce significant cytotoxic effects on a murine macrophage cell line (15). Sprague Dawley rats were administered 0.3, 1.0, and $3.0 \mathrm{~g} / \mathrm{kg}$ Angelicae dahuricae radix compound corresponding to the unprocessed drug of $2.78,9.28$, and $27.8 \mathrm{~g} / \mathrm{kg}$ by per os once a day for 12 weeks (16). The amounts were estimated to be equal to 30, 100 and 300 times the clinical dose, and no apparent chronic toxicity was reported during the experimental period. Similarly, the present study showed that cultures growing in the presence of Angelicae dahuricae radix showed marginal increase in cell number in the 0.1 and $1 \mu \mathrm{g} / \mathrm{ml}$ groups; however, statistically significant differences were not identified.

There is increasing interest in mesenchymal stem cells provide as they are an advantageous alternative therapeutic option for tissue regeneration in comparison to current treatment modalities (17). Previous reports showed that stem cells derived from the gingiva exhibited colony-forming abilities, plastic adherence and multilineage differentiation (osteogenic, adipogenic, chondrogenic) potency, and expressed CD44, CD73, CD90 and CD105 (18). Gingiva from the maxillofacial region may be considered as a favorable source of mesenchymal stem cells as the harvesting of stem cells from the mandible or maxilla can be performed easily under local anesthesia (19). Furthermore, harvesting stem cells from the gingiva may be more practical when compared with the bone marrow of the maxilla and mandible as it is less invasive and has less complications, including paresthesia and pain (20-22).

Within the limits of this study, Angelicae dahuricae radix at the tested concentrations did not produce statistically significant differences in the viability of stem cells derived from the gingiva.

\section{Acknowledgements}

This study was supported by the Basic Science Research Program through the National Research Foundation of Korea 
(NRF) funded by the Ministry of Science, ICT \& Future Planning (grant no. NRF-2014R1A1A1003106).

\section{References}

1. Wang Y, Cao SE, Tian J, Liu G, Zhang X and Li P: Auraptenol attenuates vincristine-induced mechanical hyperalgesia through serotonin 5-HT1A receptors. Sci Rep 3: 3377, 2013.

2. Zhou RH: Resource science of Chinese medicinal materials. China Medical \& Pharmaceutical Sciences Press, Beijing, pp19-32, 1993.

3. Lee H, Lee JK, Ha H, Lee MY, Seo CS and Shin HK Angelicae dahuricae radix inhibits dust mite extract-induced atopic dermatitis-like skin lesions in NC/Nga mice. Evid Based Complement Alternat Med 2012: 743075, 2012.

4. Li H, Dai Y, Zhang H and Xie C: Pharmacological studies on the Chinese drug radix Angelicae dahuricae. Zhongguo Zhong Yao Za Zhi 16: 560-562, 576, 1991 (In Chinese).

5. Kang OH, Chae HS, Oh YC, et al: Anti-nociceptive and anti-inflammatory effects of Angelicae dahuricae radix through inhibition of the expression of inducible nitric oxide synthase and NO production. Am J Chin Med 36: 913-928, 2008.

6. Yi S, Cho JY, Lim KS, et al: Effects of Angelicae tenuissima radix, Angelicae dahuricae radix and Scutellariae radix extracts on cytochrome $\mathrm{P} 450$ activities in healthy volunteers. Basic Clin Pharmacol Toxicol 105: 249-256, 2009.

7. Zhao G, Peng C, Du W and Wang S: Pharmacokinetic study of eight coumarins of Radix Angelicae dahuricae in rats by gas chromatography-mass spectrometry. Fitoterapia 89: 250-256, 2013.

8. Tang W and Eisenbrand G: Angelica spp In: Chinese drugs of plant origin, chemistry, pharmacology and use in traditional and modern medicine. Springer, Berlin, pp113-125, 1992.

9. Szucs V and Freitag R: Comparison of a three-peptide separation by capillary electrochromatography, voltage-assisted liquid chromatography and nano-high-performance liquid chromatography. J Chromatogr A 1044: 201-210, 2004.

10. Xie Y, Chen Y, Lin M, Wen J, Fan G and Wu Y: High-performance liquid chromatographic method for the determination and pharmacokinetic study of oxypeucedanin hydrate and byak-angelicin after oral administration of Angelica dahurica extracts in mongrel dog plasma. J Pharm Biomed Anal 44: 166-172, 2007.

11. Zheng X, Zhang X, Sheng X, et al: Simultaneous characterization and quantitation of 11 coumarins in Radix Angelicae dahuricae by high performance liquid chromatography with electrospray tandem mass spectrometry. J Pharm Biomed Anal 51: 599-605, 2010.
12. Liu R, Li A and Sun A: Preparative isolation and purification of coumarins from Angelica dahurica (Fisch. ex Hoffn) Benth, et Hook. $\mathrm{f}$ (Chinese traditional medicinal herb) by high-speed counter-current chromatography. J Chromatogr A 1052: 223-227, 2004.

13. Zhu M, Liang XL, Zhao LJ, et al: Elucidation of the transport mechanism of baicalin and the influence of a Radix Angelicae dahuricae extract on the absorption of baicalin in a Caco-2 cell monolayer model. J Ethnopharmacol 150: 553-559, 2013.

14. Thanh PN, Jin W, Song G, Bae K and Kang SS: Cytotoxic coumarins from the root of Angelica dahurica. Arch Pharm Res 27: 1211-1215, 2004.

15. Kang OH, Lee GH, Choi HJ, et al: Ethyl acetate extract from Angelica dahuricae Radix inhibits lipopolysaccharide-induced production of nitric oxide, prostaglandin E2 and tumor necrosis factor-alpha via mitogen-activated protein kinases and nuclear factor-kappaB in macrophages. Pharmacol Res 55: 263-270, 2007.

16. Zhao W, Cao Y and Liu J: Research on chronic toxicology of Compound Radix Angelicae dahuricae capsule. J Shaxi Med Univ 37: 160-165, 2006.

17. Moshaverinia A, Chen C, Xu X, et al: Bone regeneration potential of stem cells derived from periodontal ligament or gingival tissue sources encapsulated in RGD-modified alginate scaffold. Tissue Eng Part A 20: 611-621, 2014.

18. Jeong SH, Lee JE, Jin SH, Ko Y and Park JB: Effects of Asiasari radix on the morphology and viability of mesenchymal stem cells derived from the gingiva. Mol Med Rep 10: 3315-3319, 2014.

19. Tomar GB, Srivastava RK, Gupta N, et al: Human gingiva-derived mesenchymal stem cells are superior to bone marrow-derived mesenchymal stem cells for cell therapy in regenerative medicine. Biochem Biophys Res Commun 393: 377-383, 2010.

20. Park JB, Kim YS, Lee G, Yun BG and Kim CH: The effect of surface treatment of titanium with sand-blasting/acid-etching or hydroxyapaptite-coating and application of bone morphogenetic protein-2 on attachment, proliferation and differentiation of stem cells derived from buccal fat pad. Tissue Eng Regen Med 10: 115-121, 2013.

21. Fournier BP, Larjava H and Häkkinen L: Gingiva as a source of stem cells with therapeutic potential. Stem Cells Dev 22: 3157-3177, 2013.

22. Yang H, Gao LN, An Y, et al: Comparison of mesenchymal stem cells derived from gingival tissue and periodontal ligament in different incubation conditions. Biomaterials 34: 7033-7047, 2013. 